\title{
CUP and DISC OPTIC Segmentation Using Optimized Superpixel Classification for Glaucoma Screening
}

\author{
Prof. Vaishali Sarangpure \\ Assistant Professor, Department of Computer Science and Engineering \\ K.D.K. Women's College India \\ Vaishali.sarangpure@gmail.com
}

\begin{tabular}{|c|c|}
\hline Article History & Abstract \\
\hline $\begin{array}{l}\text { Article Submission } \\
15 \text { February } 2014 \\
\text { Revised Submission } \\
27 \text { May } 2014 \\
\text { Article Accepted } \\
28 \text { July } 2014 \\
\text { Article Published } \\
30 \text { September } 2014\end{array}$ & $\begin{array}{l}\text { Glaucoma, an incurable disease related to eyes which results in loss of the vision. } \\
\text { Identifying this disease within in a proper period of time is most important, since it } \\
\text { cannot be cured. The important aspect of this paper is to detect glaucoma at initial } \\
\text { stages. Segmentation in the optic disc necessitates the differentiation of each super } \\
\text { pixel by employing Histograms, centre surround statistics. Information location in } \\
\text { merged with the above methods in increasing the performance of optic cup } \\
\text { segmentation. Optic disc and optic cups are employed to evaluate cup to disc ratio of } \\
\text { the disease identified. Neural network is used to extract the patterns and also to } \\
\text { detect glaucomatous cells that are too complex to be noticed by either humans or } \\
\text { other computer techniques. } \\
\text { Keywords: Glaucomatous, peri papillary atrophy. }\end{array}$ \\
\hline
\end{tabular}

\section{Introduction}

Glaucoma is a chronic eye disease in which the nerve of the eye is degraded successively. It results in blindness; statistics says that 80 million people may be attacked by this disease in future. Glaucoma is also called as silent thief of sight since the symptoms will be known at its advanced stage [1]. The progression of the disease can be minimised by providing treatment at its initial stages, so it is necessary to detect glaucoma at its initial stage. The patients are unaware of the disease and they get knowledge about it, when it is in its advanced stage. The major symptom of glaucoma is the vision loss, it cannot be cured. Here, we discuss some methods to identify glaucoma [2].

IOP also called as air puff test which is not responsive in identifying glaucoma since glaucoma may generate intraocular pressure or not. This disease is tested only in famous hospitals that needs advance equipments. Therefore, it is not suitable for screening. Analyzing nerve head degradation is an important process and it is more helpful than IOP measurement or screening glaucoma [3][4]. It can also be tested by professionals who are trained but it is subjective. It has the disadvantage of taking more time and cost is high. So, we go for dynamic strategy for analyzing the disease. The major important strategy in analyzing optic nerve is to employ selection of features of the image and identifying the difference between damaged cells and healthy cells. It is not that easy as we say, but more challenging. Parameters like CDR, PPR etc are taken into consideration. Larger CDR observations identify the presence of glaucoma. Neural network is also studied in this paper.

\section{Related Work}

In identifying glaucoma we employ three methods which are discussed below. tonometry and ophthalmoscopy are the eye tests to check Glaucoma. Goniometry is an eye test which calculates the pressure of the inner eye. Generally we use drops to make the eye senseless [5]. Next, a trained professional will employ equipment known as goniometry that identifies the pressure of the eye. Generally it varies between $10 \mathrm{mmHg}$ and $22 \mathrm{mmHG}$. Ophthalmoscopy is another test employed to scan the inner side of the eye that is a nerve head. A 
professional or a doctor enlarges the inner side of the eye by employing ophthalmoscope in a closed dark room. It helps him to clearly identify the shape, colour of the nerve. Whenever pressure level is unusual, later the tests will be conducted such as perimetry and Gonioscopy [6]. Perimetry test also known as visual field test, in which the glaucoma affected person's head is said to see straight. Thus, a light moves his/her circumferential vision and identify the values [7][8].

Gonioscopy is an easy test where the feels better (without pain). It identifies the angle at which iris meets cornea that is an open angle or closed angle disease is available. Disadvantage of this system is that the analyzing of Optic nerve will be performed by a doctor[9]. Moreover, this analyzing is personalized, takes more time and high cost. Hence, we go for dynamic tests. The major strategy for this analyzing is to employ features of the image identification with unhealthy and healthy cells. They are calculated at the level of the image itself. It is not so easy and also challenging. Another strategy is to go for medical indicators. Several glaucoma features are taken into consideration such as CDR, PPA. Analyzing manually the image features takes more time. Also, disadvantage is that exactness varies between the several professionals [10].

\section{Proposed Data path Designs}

In glaucoma screening, Localization and segmentation of disc is an important feature in several diagnosis devices. Localization identifies disc pixel(centre of disc). Then segmentation approximates the boundary of the disc which is very difficult and challenging because of blood vessels, variations in the disease, image variations etc. The fig.1 shows that the input image is generated using super pixel algorithm and then its five channels such as red, green, blue, hue and saturation are processed from which its features are extracted and classified. Then the image is deformed to its original form.

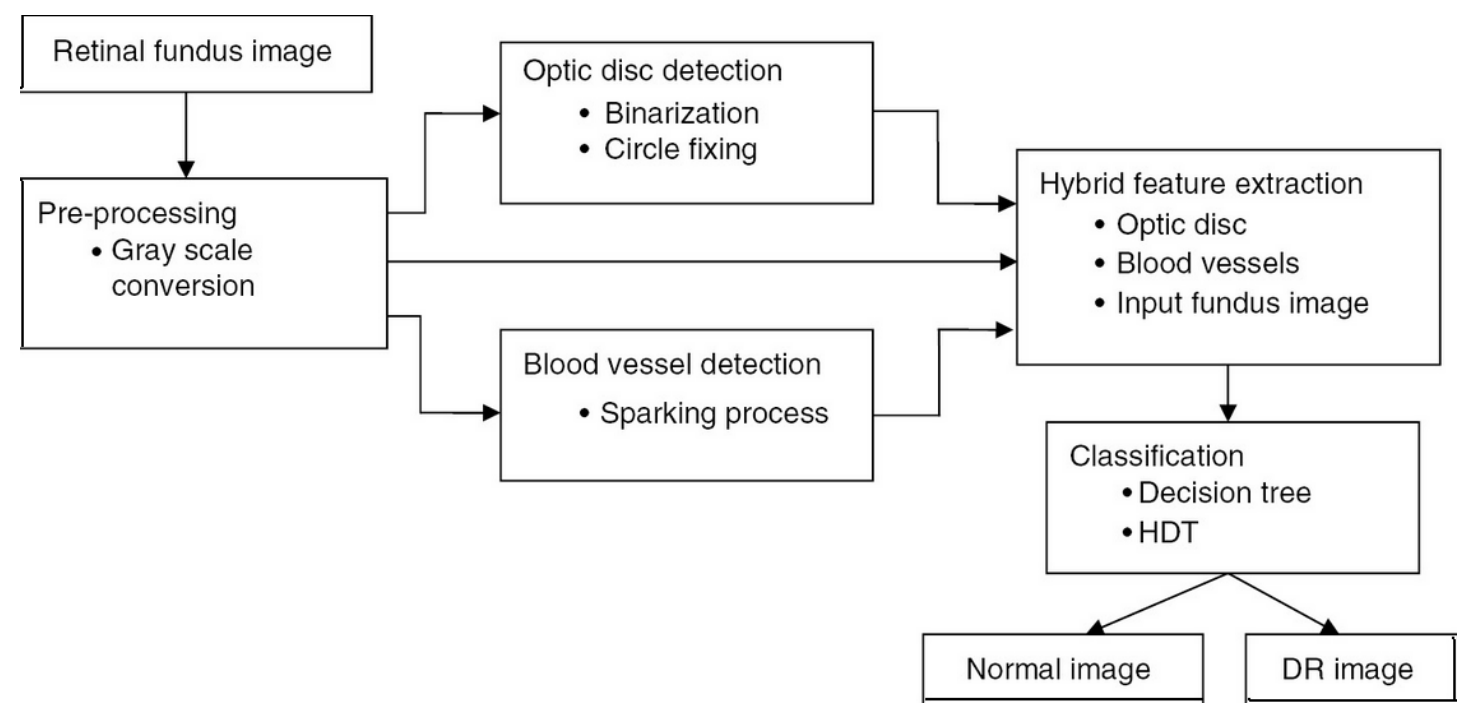

Fig 1: Proposed method using super pixel algorithm

Important risk is to segment the cup whether the pallor is weak or usual. During this case, changes in the intensity or approximating the cup boundaries lack. These can be seen at different locations in the disc and only some points identify the boundary of the cup. A 2-D image where no depth is present is a difficult task to identify the boundary of the cup. Here, cup region is the pallor which is the high colour contrast in the disc. The other one is the bends in the blood vessels. Retinal nerves combines to form an optic cup shaped -location known as cup. Another challenging task is the disc segmentation of the optic. In specific, same regions identified at the boundary are the concern to meet disc segmentation. The five channels such as red, green, blue, 
hue and saturation are processed from which its features are extracted and classified. Then the image is smoothed such that ellipse fitting is done where the cells get affected.

\section{Simulation Results}

Simulations are performed using MATLAB. After obtaining the disc and cup, various features can be computed. It follows the clinical convention to compute the CDR. CDR is an important indicator for glaucoma screening. The computed CDR is used for glaucoma screening. When CDR is greater than a threshold, it is glaucomatous, otherwise, healthy. Different components are evaluated after the disc and cup segmentation. We go for clinical It helps in evaluating CDR. It is a major identifier for screening a glaucoma. Then, It is adopted for glaucoma screening. If it is more than threshold, glaucomatous is confirmed. The figure 2 shows the various source images of eye with their estimated portion of the presence of glaucoma.

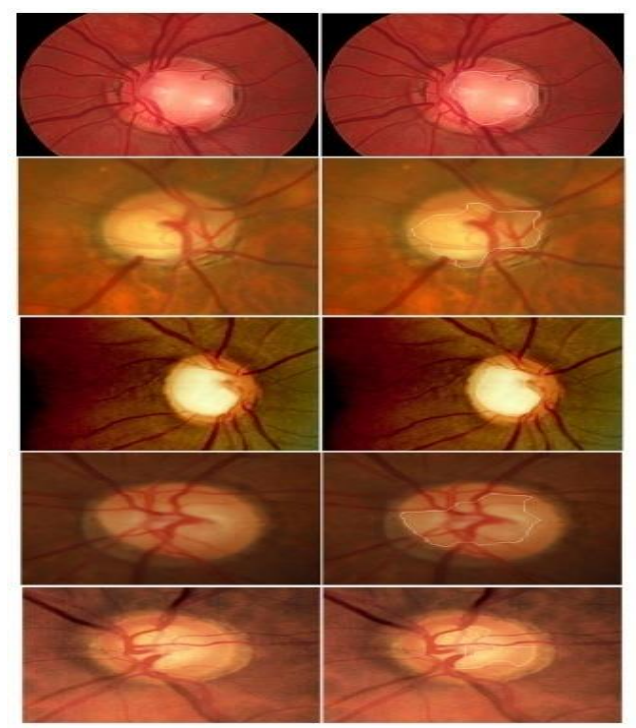

Fig 2: Source images with their Gradient estimated Glaucoma region

Disadvantages of classification of pixel techniques can be controlled by super pixel classification technique which merges with deformable model. Local image features are evaluated by super pixels which are local and coherent. It also identifies more repetitions in the image which also decreases difficulty of processing the next steps. Classification of super pixel employs introduction of OD boundary. Deformable model helps in fine tuning the OD boundary. It also evaluates self-assessment of OD. It is a major block that is rarely studied in OD segmentation. But we go for dynamic segmentation method for image features.

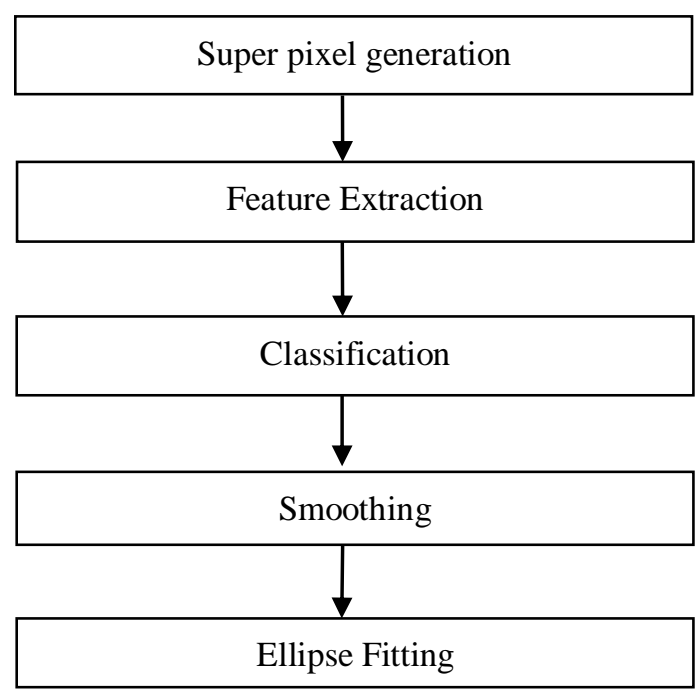

Fig 3: Flow diagram to show Glaucoma region 
The OD segmentation is dependent on Super pixel classification merges into the production of super pixels, which evolves the image feature from them. Also, the evaluation of self-assessment reliability. This step is employed to classify the image into many super pixels.

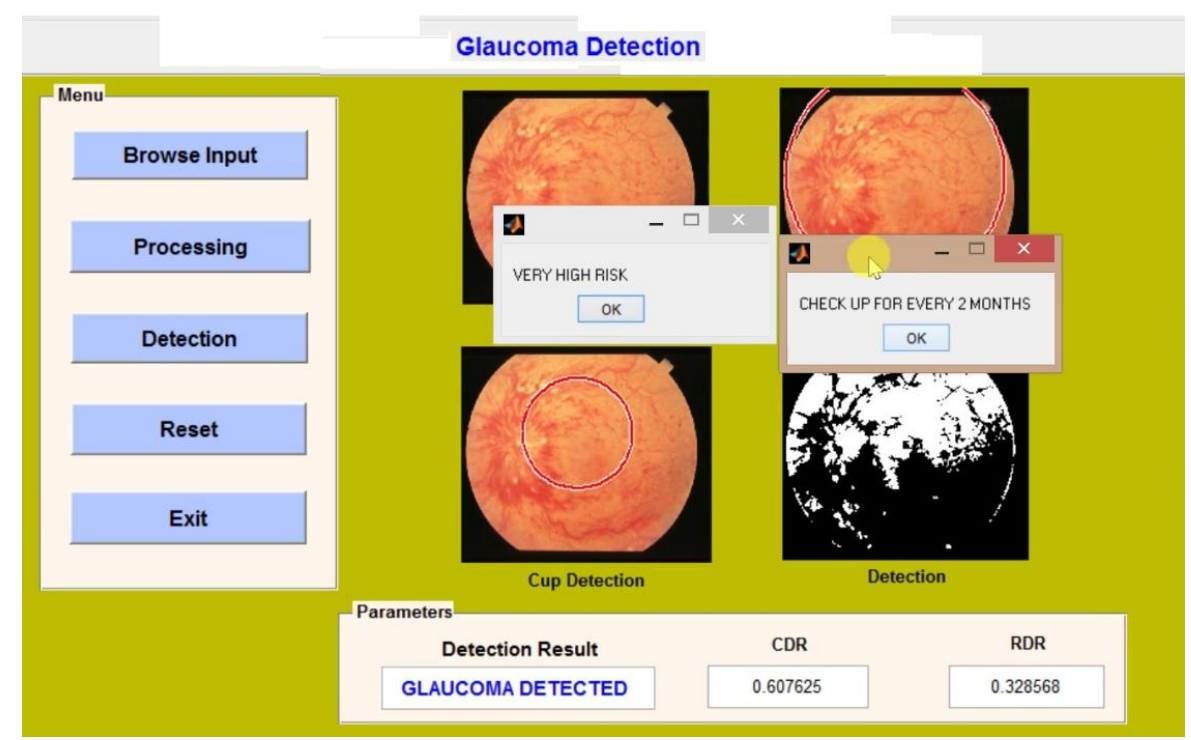

Fig 4: Simulation window showing glaucoma detection

\section{Conclusion}

For cell classification between healthy and glaucomatous cell neural network is used in this project and the algorithm used is feed forward algorithm. The output is displayed as a prompt or a message box with a detail whether the eye is affected by glaucoma or not. In this work, glaucoma is detected by using super pixel algorithm that optic disc and optic cup are separately segmented and processed. It detects glaucoma in initial stage and by using Super pixel algorithm it is processed faster than other methods. For neural network, the algorithm used is feed forward by using this glaucomatous and healthy cells are classified. The future enhancement of this project can be done by using the same technique for detection of other chronic eye diseases. It can also be enhanced by displaying the stages of the disease along with whether it is healthy or not.

\section{References}

[1]

[2] Bock. R, Meier. J, Nyl. L.G and Michelson. G (2010), 'Glaucoma risk index: Automated glaucoma detection from colour fundus images', Medical Image Analysis, Vol. 14, pp.No. 471-481.

[3] Duan. L, Tsang. I. W, and Xu. D (2012), 'Domain transfer multiple kernel learning', IEEE Transaction Pattern Analysis Machine Intelligence, Vol. 34, No. 3, pp.No.465-479.

[4] Joshi. G. D, Sivaswamy. J and Krishnadas. S. R (2011), 'Optic disk and cup segmentation from monocular colour retinal images for glaucoma assessment', IEEE Transaction Medical Image, Vol.30, No.6, pp.No.1192-1205, Jun.

[5] Shaji.A, Hugh Fedrick.S, Achanta.R Smith.K, Lucchi.A, Fua (2012), 'Slic superpixels compared to state-of-the-art superpixel methods', IEEE Transaction Pattern Analysis Machine Intelligence, Vol. 34, No.11, pp.No.2274-2281.

[6] Veksler.O, Boykov.Y (2012), 'Superpixels and supervoxels in an energy optimization framework', in Europe Conference Computer Vision, Vol. 5, pp. No.211-224.

[7] N. Noor, N. Khalid and N. Ariff, "Optic cup and disc color channel multi-thresholding segmentation," 2013 IEEE International Conference on Control System, Computing and Engineering, Mindeb, 2013, pp. 530-534, doi: 10.1109/ICCSCE.2013.6720022.

[8] J. Cheng et al., "Superpixel Classification Based Optic Disc and Optic Cup Segmentation for Glaucoma Screening," in IEEE Transactions on Medical Imaging, vol. 32, no. 6, pp. 1019-1032, June 2013, doi: 10.1109/TMI.2013.2247770. 
[9] F. Yin et al., "Automated segmentation of optic disc and optic cup in fundus images for glaucoma diagnosis," 2012 25th IEEE International Symposium on Computer-Based Medical Systems (CBMS), Rome, 2012, pp. 1-6, doi: 10.1109/CBMS.2012.6266344.

[10] J. B. Jonas, A. Bergua, P. Schmitz-Valckenberg, K. I. Papastathopoulos and W. M. Budde, "Ranking of optic disc variables for detection of glaucomatous optic nerve damage", Invest. Ophthalmol. Vis. Sci., vol. 41, no. 7, pp. 1764-1773, 2000

[11] K. Lee, M. Niemeijer, M. K. Garvin, Y. H. Kwon, M. Sonka and M. D. Abràmoff, "Segmentation of the optic disc in 3-D OCT scans of the optic nerve head", IEEE Trans. Med. Imag., vol. 29, no. 1, pp. 159-168, Jan. 2010.

[12] G. D. Joshi, J. Sivaswamy and S. R. Krishnadas, "Optic disk and cup segmentation from monocular color retinal images for glaucoma assessment", IEEE Trans. Med. Imag., vol. 30, no. 6, pp. 1192-1205, Jun. 2011 